\title{
Efficacy of the MEK Inhibitor Cobimetinib and its Potential Application to Colorectal Cancer Cells
}

\author{
Shu Gonga,b Dongsheng Xua Jialin Zhu ${ }^{a} \quad$ Fangdong Zou ${ }^{a} \quad$ Rui Peng ${ }^{a}$ \\ ${ }^{a}$ College of Life Sciences, Sichuan University, Chengdu, bDepartment of Basic Medicine, Zhaoqing \\ Medical College, Zhaoqing, P.R. China
}

\author{
Key Words \\ Cobimetinib • Colorectal Cancer • Cancer therapy • Thymidylate synthetase
}

\begin{abstract}
Background/Aims: Mutations in the Ras/Raf/MEK/ERK pathway are detected in 50\% of colorectal cancer cases and play a crucial role in cancer development and progression. Cobimetinib is a MEK inhibitor approved for the treatment of advanced melanoma and inhibits the cell viability of other types of cancer cells. Methods: HCT116 colorectal cancer cells were treated with cobimetinib, and MTT assay, colony formation assay, and flow cytometry were used to evaluate cell viability, cell cycle, and apoptosis, respectively. The expression of genes associated with the cell cycle and apoptosis were evaluated by quantitative real-time PCR and western blotting. To explore use of cobimetinib in colorectal cancer treatment and further understand its mechanisms, RNA-seq technology was used to identify differentially expressed genes (DEGs) between cobimetinib-treated and untreated HCT116 cells. Furthermore, we compared these DEGs with Gene Expression Omnibus data from colorectal cancer tissues and normal colonic epithelial tissues. Results: We found that cobimetinib not only inhibited cell proliferation but also induced G1 phase arrest and apoptosis in HCT116 colorectal cancer cells, suggesting that cobimetinib may useful in colorectal cancer therapy. After cobimetinib treatment, 3,495 DEGs were obtained, including 2,089 upregulated genes and 1,406 downregulated genes, and most of these DEGs were enriched in the cell cycle, DNA replication, and DNA damage repair pathways. Our results revealed that some genes with high expression in colorectal cancer tissues were downregulated by cobimetinib in HCT116 cells, including CCND1, E2F1, CDC25C, CCNE2, MYC, and PCNA. These genes have vital roles in DNA replication and the cell cycle. Furthermore, genes with low expression in colorectal cancer tissues were upregulated by cobimetinib, including PRKCA, PI3K, RTK, and PKC. Based on our results, the PKC and PI3K pathways were activated after cobimetinib treatment, and inhibition of these two pathways can increase the cytotoxicity of cobimetinib in HCT116 cells. Notably, cobimetinib appeared to enhance the efficacy of 5-fluorouracil (5-FU) by decreasing
\end{abstract}

S. Gong and D. Xu contributed equally to this work.

Rui Peng

and Fangdong Zou
College of Life Sciences, Sichuan University

Chengdu (China), Tel. +86 28 85412805, Fax +86 2885412805

E-Mail pengrui@scu.edu.cn, fundzou@scu.edu.cn

\section{KARGER}


TYMS expression, high expression of which is responsible for 5-FU resistance in colorectal cancer. Conclusions: Our results suggest the potential use of cobimetinib in colorectal cancer therapy.

(C) 2018 The Author(s)

Published by S. Karger AG, Basel

\section{Introduction}

The Ras/Raf/MEK/ERK pathway regulates cell proliferation, survival, differentiation, and angiogenesis, and plays a critical role in tumorigenesis and development $[1,2]$. Mutations in the composition of this pathway, especially the acquired mutation of K-Ras or $\mathrm{B}$-Raf, can result in its hyperactivation, which is highly correlated with tumorigenesis [3]. The occurrence of colorectal cancer is accompanied by mutations in many genes, though K-Ras is the most common type of mutation [4]. As a downstream molecule of Ras, Raf mutations also play an important role in the pathogenesis of colorectal cancer [5]. Together, the Ras/ Raf/MEK/ERK pathway is an important target for the discovery of anticancer drugs to treat colorectal cancer.

Thus far, a growing number of small molecule inhibitors have been used in clinical treatment. They can effectively prolong the survival of patients with colorectal cancer, especially metastatic colorectal cancer [6]. Regorafenib is the first small molecule inhibitor approved by the FDA for the treatment of metastatic colorectal cancer, and it targets the Ras/ Raf/MEK/ERK signaling pathways [7]. Zhang et al. reported that regorafenib inhibited the growth of colorectal cancer by inducing PUMA-mediated apoptosis [8]. However, to date, there are only two MEK-specific inhibitors approved by the FDA for clinical use, namely, trametinib and cobimetinib.

Cobimetinib (GDC-0973) is a potent and selective small molecule inhibitor that specifically targets MEK1/2. Cobimetinib selectively inactivates MEK kinase, thereby blocking the transduction of Ras/Raf pathway signaling [9]. In 2015, the FDA approved cobimetinib for BRAF-mutated melanoma in combination with vemurafenib, a BRAF inhibitor [10]. It has been shown that cobimetinib inhibits the cell viability of many cancer cells, including colorectal cancer $[11,12]$. Regarding safety, cobimetinib treatment had a well-tolerated profile, even at the maximum administered oral daily doses of $60 \mathrm{mg}$ (Clinical Trial: NCT01988896) [13]. However, its effect and mechanism of action on colon cancer is still uncertain.

In this study, we found that cobimetinib could induce G1 arrest and apoptosis in HCT116 colorectal cancer cells, which is consistent with a previous study [12]. To further investigate the mechanisms of cobimetinib on colorectal cancer, RNA-seq technology was employed to explore differentially expressed genes (DEGs) after cobimetinib treatment. Through a combined analysis with Gene Expression Omnibus (GEO) data from colorectal cancer tissues and normal colonic epithelial tissues, we found that cobimetinib can induce the activation of PKC and PI3K pathways and enhance the efficacy of 5-fluorouracil (5-FU) by decreasing TYMS expression. Our results reveal a high potential for using cobimetinib in colorectal cancer treatment.

\section{Materials and Methods}

\section{Cell culture and treatment}

The human colorectal cancer cell lines HCT116, RKO, HT-29, SW480, and DLD-1 were obtained from the Cell Library of the Chinese Academy of Sciences. HCT116 p53\% and HCT116 p $21^{\%}$ were described previously [14] and obtained from Dr. Bert Vogelstein (Sidney Kimmel Comprehensive Cancer Center, Johns Hopkins University, Baltimore, MD, USA). All cell lines were grown in high-glucose Dulbecco's modified Eagle medium(HyClone, South Logan, UT, USA) supplemented with $10 \%(v / v)$ defined fetal bovine serum (Gibco, Gaithersburg, MD, USA), $100 \mathrm{U} / \mathrm{mL}$ penicillin, and $100 \mu \mathrm{g} / \mathrm{mL}$ streptomycin (HyClone) and then maintained in a humidified incubator with $5 \% \mathrm{CO}_{2}$ at $37^{\circ} \mathrm{C}$. Cobimetinib, VS-5584, and Go 6983 were 


\section{Cellular Physiology Cell Physiol Biochem 2018;47:680-693 \begin{tabular}{l|l} 
DOI: 10.1159/000490022 & O 2018 The Author(s). Published by S. Karger AG, Basel \\
www.karger.com/cpb
\end{tabular}}

Gong et al.: Potential of Cobimetinib in Colorectal Cancer

purchased from Selleck Chemicals (Houston, TX, USA) and dissolved in dimethyl sulfoxide (DMSO; SigmaAldrich, St. Louis, MO, USA) at stock concentration. Then, 5-FU (Sigma-Aldrich) was dissolved in phosphatebuffered saline (PBS, pH 7.4) at stock concentration. Drugs at stock concentrations were stored in the dark at $-20^{\circ} \mathrm{C}$. For drug treatment assays, the cells were seeded into 6-well or 12-well plates, and then treated with concentrations prepared by appropriate dilution with the complete culture medium when cells reached about $30 \%$ confluence.

\section{Cell viability assay (MTT assay) and combination index determination}

Cell viability was evaluated by MTT assays. HCT116 colorectal cancer cells were trypsinized and seeded into 96-well plates at a density of 8, 000 cells per well. After overnight incubation, cells were treated with different concentrations of the agents for $48 \mathrm{~h}$, and the DMSO-treated (0.1\%) cells were regarded as the control group. Then, $10 \mu \mathrm{L}$ MTT solution $(5 \mathrm{mg} / \mathrm{mL})$ was added to each well and incubated for $4 \mathrm{~h}$. Afterwards, the supernatant was replaced with $100 \mu \mathrm{L}$ DMSO to dissolve the insoluble formazan crystals. Finally, the absorbance was detected at $490 \mathrm{~nm}$ or $570 \mathrm{~nm}$. The growth inhibition (\%) was determined using the following equation: Growth inhibition (\%) = 1 - (Mean of absorbance of treated group) / (Mean of absorbance of control group)] $\times 100 \%$. A combination index (CI) was calculated via the Chou-Talalay method [15]. The synergism of the two drugs was evaluated by the CI, where CI > 1 indicates antagonism, CI $=1$ indicates additivity, and $\mathrm{CI}<1$ indicates synergism.

\section{Colony formation assay}

After drug treatment, the cells were seeded into 6-well plates and then incubated at $37^{\circ} \mathrm{C}$ with $5 \% \mathrm{CO}_{2}$ for 2 weeks until visible clones appeared. Then, the cells were carefully washed twice with PBS, fixed with methyl alcohol for $20 \mathrm{~min}$, and stained with $0.1 \%$ crystal violet solution for $30 \mathrm{~min}$.

\section{Western blot analysis}

After drug treatment, the cells were gathered and lysed with $2 \times$ sample buffer $(10 \%$ sodium dodecyl sulfate [SDS], Tris- $\mathrm{HCl} \mathrm{pH} \mathrm{6.8,} \mathrm{glycerol,} 0.5 \%$ bromophenol blue, and $\beta$-mercaptoethanol). The protein samples were boiled at $96^{\circ} \mathrm{C}$ for $10 \mathrm{~min}$ and stored at $-20^{\circ} \mathrm{C}$. Then, the samples were isolated by SDS polyacrylamide gel electrophoresis and subsequently transferred onto polyvinylidene fluoride membranes (Millipore, Billerica, MA, USA). The non-specific sites of the membrane were blocked using 5\% skim milk $(w / v)$ in tris-buffered saline with $0.1 \%$ Tween-20 (TBST) for $1.5 \mathrm{~h}$ at room temperature and incubated at $4{ }^{\circ} \mathrm{C}$ with primary antibodies overnight. The primary antibodies were anti-p21 (1:2000, Proteintech, Chicago, IL, USA), anti-p53 (1:2000, Proteintech), anti-cyclin D1 (1:2000, Zen BioScience, Chengdu, China), anti-cyclin E (1:2000, Zen BioScience), anti-TYMS (1:2000, Zen BioScience), anti- $\beta$-actin (1:5000, Proteintech), and antiPARP (1:2000, Zen BioScience). The membranes were washed with TBST three times and then incubated with corresponding secondary antibodies for $1.5 \mathrm{~h}$ at room temperature. Finally, the blots were visualized using Immobilon Western Chemiluminescent HRP Substrate (Millipore).

\section{Quantitative real-time PCR ( $q$ RT-PCR)}

Total RNA of the cells was isolated using the RNAiso Plus kit (TaKaRa, Dalian, China) according to the manufacturer's protocol and was stored at $-80^{\circ} \mathrm{C}$. The PrimeScript ${ }^{\mathrm{TM}} \mathrm{RT}$ reagent Kit with gDNA Eraser (TaKaRa) was used to synthesize first-strand cDNAs. Subsequently, target gene expression was examined using EvaGreen $2 \times$ qPCR MasterMix-No Dye (abm, Richmond, BC, Canada) by qRT-PCR. The relative expression levels of the target genes were analyzed according the $2^{-\Delta \Delta C t}$ method. The GAPDH gene was used as an internal control to standardize variance among the target gene expression levels. All sample measurements were conducted in triplicate. qRT-PCR primers were synthesized by BGI TECH (Beijing, China), and the primer sequences are listed in Table 1.

\section{Cell cycle arrest assay}

Colorectal cancer cells were plated into 6- or 12-well plates and treated with various concentrations of reagents for $48 \mathrm{~h}$. Then, the cells were harvested, washed with PBS, centrifuged and fixed in cold $70 \%$ ethanol overnight at $4^{\circ} \mathrm{C}$. After fixation, the samples were washed with PBS. Afterward, the cells were resuspended in PBS containing $10 \mathrm{mg} / \mathrm{mL}$ propidium iodide (PI; Sigma-Aldrich) and RNase A (100 mg/ $\mathrm{mL}$, Solarbio, Beijing, China) and incubated for 30 min under darkness at room temperature. Finally, the 


\section{Cellular Physiology Cell Physiol Biochem 2018;47:680-693 \begin{tabular}{l|l} 
and Biochemistry Published on/me:IVlay Z5, 2018 & $\begin{array}{l}\text { ( ) 2018 The Author(s). Published by S. Karger AG, Basel } \\
\text { www.karger.com/cpb }\end{array}$
\end{tabular} \\ Gong et al.: Potential of Cobimetinib in Colorectal Cancer}

samples were analyzed using the BD FACScalibur flow cytometer (BD Biosciences, Franklin Lakes, NJ, USA).

Cell apoptosis assay

Cell apoptosis was examined using the annexin V-fluorescein isothiocyanate (FITC) staining (Annexin-FITC Apoptosis Detection Kit, Biotool, Houston, TX, USA) with flow cytometry. Colorectal cancer cells were seeded into 6or 12-well plates and treated with various concentrations of reagents for $48 \mathrm{~h}$. Then, the cells were harvested, washed with PBS, centrifuged, and resuspended in $1 \times$ binding buffer. Next, the cells were stained with annexin V-FITC and PI for $10 \mathrm{~min}$ in the dark at room temperature. Finally, the samples were analyzed using the BD FACScalibur flow cytometer (BD Biosciences).

Alternatively, Hoechst 33258 staining was also employed to evaluate cell apoptosis. Briefly, the cells were harvested, washed with PBS, and centrifuged. Then, the cells were incubated with Hoechst 33258 staining solution (300 $\mu \mathrm{L}$ PBS, $20 \mu \mathrm{L}$ 10\% NP-40, $36 \mu \mathrm{L} \mathrm{37 \%}$ formaldehyde, 4 $\mu \mathrm{L} 1 \mathrm{mg} / \mathrm{mL}$ Hoechst 33258) for $30 \mathrm{~min}$ in the dark at room temperature. Finally, apoptosis was calculated under fluorescence microscopy.

\section{RNA-Seq and bioinformatics analysis}

The HCT116 cells were seeded into 6-well plates and treated with $1 \mu \mathrm{M}$ cobimetinib for $24 \mathrm{~h}$ or $36 \mathrm{~h}$. Then, the cells were collected by centrifugation and resuspended in RNAiso Plus (TaKaRa). Transcriptomes were sequenced on the Illumina HiSeq platform (Illumina, San Diego, CA, USA). Thresholds of Q value $<0.05$ and $\mid \log _{2}$ (fold change) | > 1 were set for screening DEGs. Gene functional annotations were based on the genome database and mapped onto Gene Ontology (GO) terms in the GO database (http://www.geneontology.org/). Pathway analysis was based on the pathways available within the Kyoto Encyclopedia of Genes and Genomes (KEGG) database. The gene expression data for colorectal cancer tissues and normal colonic epithelial tissues were downloaded from the GEO database with the accession number GSE21815. We explored the intersection of genes between DEGs from RNA-seq and DEGs from CEO data. Then, the functions of these intersectional genes were annotated by KEGG. The raw reads are available from the NCBI Sequence Read Archive under accession numbers SRR5934059-SRR5934061, and the BioProject accession number is PRJNA398173.

\section{Statistical analysis}

All experiments were performed in triplicate and data were expressed as the mean \pm standard deviation (SD). Student's $t$-tests were used to compare those data. Differences with $p<0.05$ were regarded as statistically significant.

\section{Results}

Cobimetinib inhibited the cell viability of different colorectal cancer cells

To investigate the effect of cobimetinib on colorectal cancer, five different colorectal cancer cell lines were treated with different concentrations of cobimetinib, and then cell
Table 1. Primer sequences for qRT-PCR

\begin{tabular}{|c|c|c|}
\hline Gene Names & & Primers sequences $\left(5^{\prime}-3^{\prime}\right)$ \\
\hline \multirow{2}{*}{ GAPDH } & Forward & TACCAAGCTTATGGACGGGTCCGGGGAGCA \\
\hline & Reverse & ATAGAATTCCGCCCATCTTCTTCCAGATGGTG \\
\hline \multirow{2}{*}{ CCNA2 } & Forward & TCACCAGACCTACCTCAAAG \\
\hline & Reverse & CATCTTAGAAAACAAAGGCAGTC \\
\hline \multirow{2}{*}{ CCNB1 } & Forward & ACACCAACTCTACAACATTACCT \\
\hline & Reverse & GGCTAAATCTTGAACTAGTGCAG \\
\hline \multirow{2}{*}{ CCNE2 } & Forward & ATACTGACTGCTGCTGCCTTG \\
\hline & Reverse & ACATTCTGAAATACTGTCCCACT \\
\hline \multirow{2}{*}{$\mathrm{CDC} 25 \mathrm{C}$} & Forward & CCCATCGTCCCTTTGGACACCCA \\
\hline & Reverse & CCTCTTCACGCAGACAGCGGCAC \\
\hline \multirow{2}{*}{ CHEK1 } & Forward & GGGATACCAGCCCCTCATACATT \\
\hline & Reverse & TTCCATTGATAGCCCAACTTCTC \\
\hline \multirow{2}{*}{$\mathrm{E} 2 \mathrm{~F} 1$} & Forward & СТСАТСССТCACCACAGATCCCA \\
\hline & Reverse & AGAAGTCCTCCCGCACATGCTCC \\
\hline \multirow{2}{*}{ MYC } & Forward & CCCTGGTGCTCCATGAGGAGACA \\
\hline & Reverse & GGGCTGTGAGGAGGTTTGCTGTG \\
\hline \multirow[t]{2}{*}{ PCNA } & Forward & GTAATGTCGATAAAGAGGAGGAAG \\
\hline & Reverse & ATACTGAGTGTCACCGTTGAAGA \\
\hline \multirow{2}{*}{ PDCD4 } & Forward & GAGACATATCTGAAGCTGAACAT \\
\hline & Reverse & TGTACTTTCTCCAGTTGACTCTA \\
\hline \multirow{2}{*}{ PRCK } & Forward & CAGCCACCATTCAAGCCCAAAGT \\
\hline & Reverse & TACGAGAACCCTTCAAAATCAGA \\
\hline \multirow[t]{2}{*}{ RB1 } & Forward & CCAGAAGCCATTGAAATCTACCT \\
\hline & Reverse & AGGGTCCAGATGATATGTTCTAATT \\
\hline & Forward & TGGAGGAGTTGCTGTGGTT \\
\hline & Reverse & TGTCGTCAGGGTTGGTTTT \\
\hline
\end{tabular}


viability was evaluated using the MTT assay. The results showed that cobimetinib decreased the cell viability of all five colorectal cancer cell lines (Fig. 1A). Furthermore, colony formation assays showed similar long-term effects (Fig. 1B and C).

\section{Cobimetinib induced G1 arrest in HCT116 cells}

Next, we explored how cobimetinib inhibited the cell viability of colorectal cancer cells. First, HCT116 cells were treated with cobimetinib at the $\mathrm{IC}_{50}$ concentration $(1 \mu \mathrm{M})$. Then, cell cycle changes were evaluated using flow cytometry after staining with PI. Cobimetinib was observed to induce cell arrest in the G1 phase (Fig. 2A). Moreover, western blots indicated that the expression levels of cyclin D1 and cyclin E were decreased and p21 levels were increased after cobimetinib treatment (Fig. 2B). Similarly, qRT-PCR results revealed that the mRNA expression of some important cell cycle-related genes, including CCND1, CCNA2, CCNE1, CCNB1, CDC25C, CHK1, E2F, Myc, PCNA, and Rb1, were decreased after cobimetinib treatment (Fig. 2C). These results suggest that cobimetinib induced G1 phase arrest by altering the expression of cell cycle-related genes in HCT116 cells.

\section{Cobimetinib induced G1 arrest in a p53/p21 independent manner in HCT116 cells}

Cobimetinib can significantly increase the expression level of p21 (Fig. 3A and B). p53 can initiate cell cycle arrest by regulating its target gene p21 or the transcription of a series of molecules. To demonstrate whether p53 was required for cobimetinib-induced upregulation of p21, p53-deficient HCT116 cells were treated using cobimetinib. The results showed that cobimetinib can increase both the mRNA and protein levels of p21 in HCT116 p53\% cells (Fig. 3C and D). Thus, p53 expression was independent of cobimetinib-induced upregulation of p21 in HCT116 cells.

To explore whether p21 or p53 was necessary for cobimetinib-induced G1 arrest, p21 and p53-deficient HCT116 cells were treated with cobimetinib. Flow cytometry revealed that cobimetinib induced G1 arrest in the absence of p21 or p53. In addition, cobimetinib can also downregulate the expression of important cycle-related genes, including CCND1, CCNA2, CCNB1, CDC25C, CHK1, E2F, MYC, PCNA, and RB1, in p21-deficient cells (p21\% HCT116), excepted for CCNE1 (Fig. 2C). These results suggest that cobimetinib induced G1 arrest in a p53/p21-independent manner in HCT116 cells.

Fig. 1. Cobimetinib inhibited the cell viability of different colorectal cancer cell lines. (A) HCT116, SW480, DLD-1, HT-29, and RKO cells were treated with different concentrations of cobimetinib $(0-1 \mu \mathrm{M})$ for $48 \mathrm{~h}$. Then cell viability was measured by MTT assays. (B and C) A colony formation assay was used to determine the long-term effect of cobimetinib. HCT116, SW480, DLD-1, HT-29, ad RKO cells were treated with 1 $\mu \mathrm{M}$ cobimetinib for $48 \mathrm{~h}$, and then 1,000 cells were seeded into 6 -well plates and incubated for 2 weeks. Colonies were then counted.

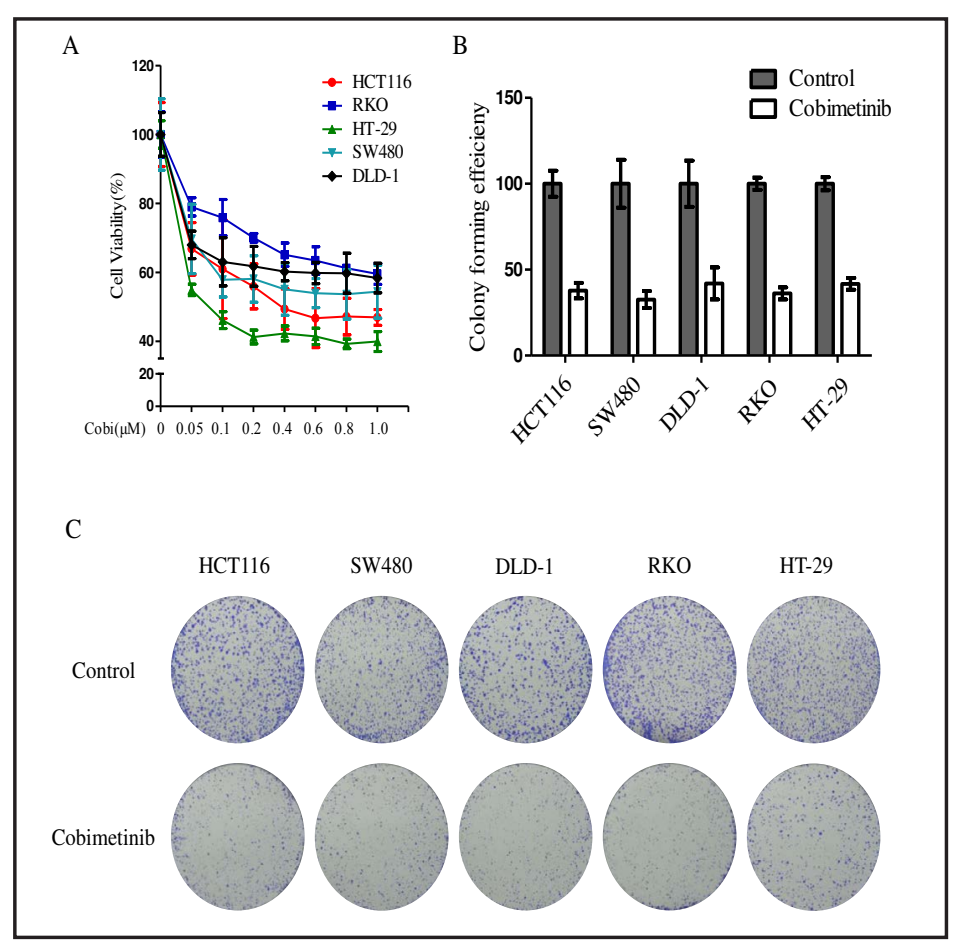


Fig. 2. Cobimetinib induced $\mathrm{G} 1$ arrest in HCT116 cells. (A and B) HCT116 cells were treated with $1 \mu \mathrm{M}$ cobimetinib for $24 \mathrm{~h}$, and then the cell cycle was evaluated using flow cytometry. The histogram provides statistical results of three independent experiments. (C) Western blots showing protein changes of p21, p53, cyclin D1, and cyclin E in HCT116 cells after treatment with $1 \mu \mathrm{M}$ cobimetinib over different timespans. (D) mRNA expression levels of cycle-related genes were evaluated by qRTPCR in parental HCT116 or p21\% HCT116 cells. $(* * p<0.01)$
A
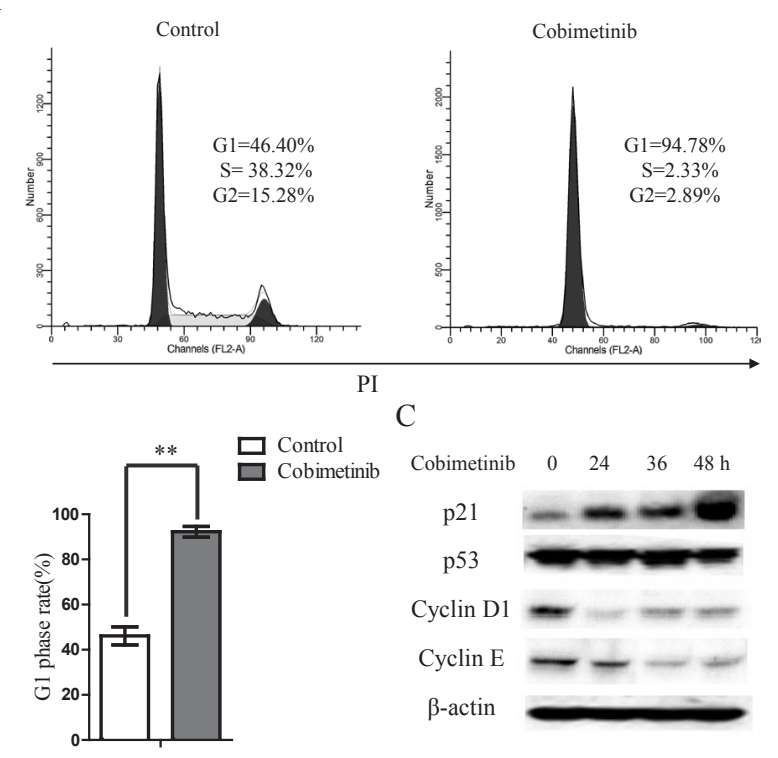

$\mathrm{D}$

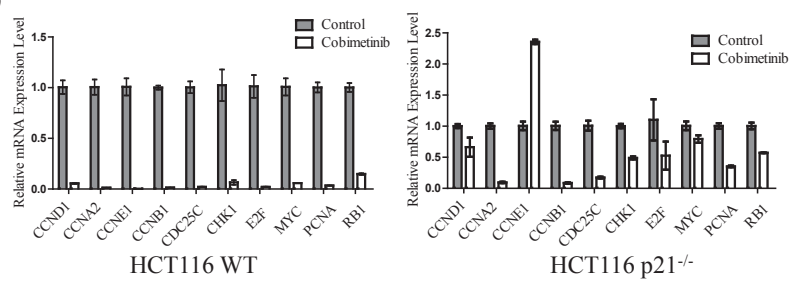

Fig. 3. Cobimetinib induced G1 arrest in a p53/p21-independent manner in HCT116 cells. (A) Relative mRNA levels of p21 were determined by q-PCR after treatment with cobimetinib $(1 \mu \mathrm{M})$ for $48 \mathrm{~h}$ in HCT116 parental and p53\% cells. (B) Western blots showing the p21 level in HCT116 parental and p53\% cells. (C) HCT116 p53\% and p $21^{\%}$ cells were treated with cobimetinib $(1 \mu \mathrm{M})$, and the cell cycle distributions were determined by flow cytometry.
A

\section{$\mathrm{B}$}

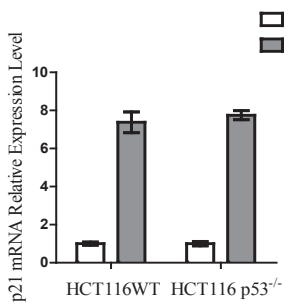

$\square$ Control
$\square$ Cobimetin

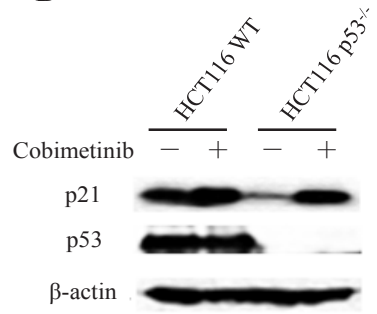

$\mathrm{C}$

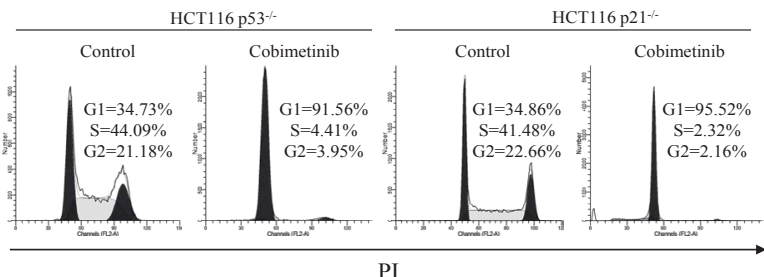

Cobimetinib induced apoptosis in HCT116 cells

It has been reported that cobimetinib can induce cell apoptosis in neuroblastoma cells [16]. To investigate whether cobimetinib could induce apoptosis in colorectal cancer cells, HCT116 was treated with cobimetinib and then stained with annexin V/PI. Flow cytometry was used to detect apoptosis induced by cobimetinib. As shown in Fig. 4A, more apoptotic cells were found after cobimetinib treatment compared with the control. The western blot result also showed that c-PARP (cleaved PARP, activated form) was increased gradually by cobimetinib (Fig. 4B). In addition, we also found that cobimetinib significantly induced apoptosis in both p21 and p53 deficient cells (p21\% HCT116 or p53\% HCT116; Fig. 4C). 
The results further suggested that cobimetinib can induce apoptosis in a p53- or p21independent manner in HCT116 cells.

\section{Comparative analysis of transcriptome data and GEO data}

To explore the application of cobimetinib on colorectal cancer and its underlying mechanisms, we employed RNA-seq technology to analyze the expression changes induced by cobimetinib. HCT116 cells were treated with cobimetinib for $24 \mathrm{~h}$ or $36 \mathrm{~h}$, and then

Fig. 4. Cobimetinib induced apoptosis in HCT116 cells. (A) Percentage of apoptotic cells was detected by annexin-V and PI staining after cobimetinib $(1 \mu \mathrm{M})$ treatment of HCT116 cells for $48 \mathrm{~h}$. (B) Western blots showing the level of c-PARP was upregulated by cobimetinib (1 $\mu \mathrm{M}$ ) treatment. (C) HCT116 p53\% and p $21^{\%}$ - cells were treated with cobimetinib $(1 \mu \mathrm{M})$, and cell apoptosis was evaluated by flow cytometry.

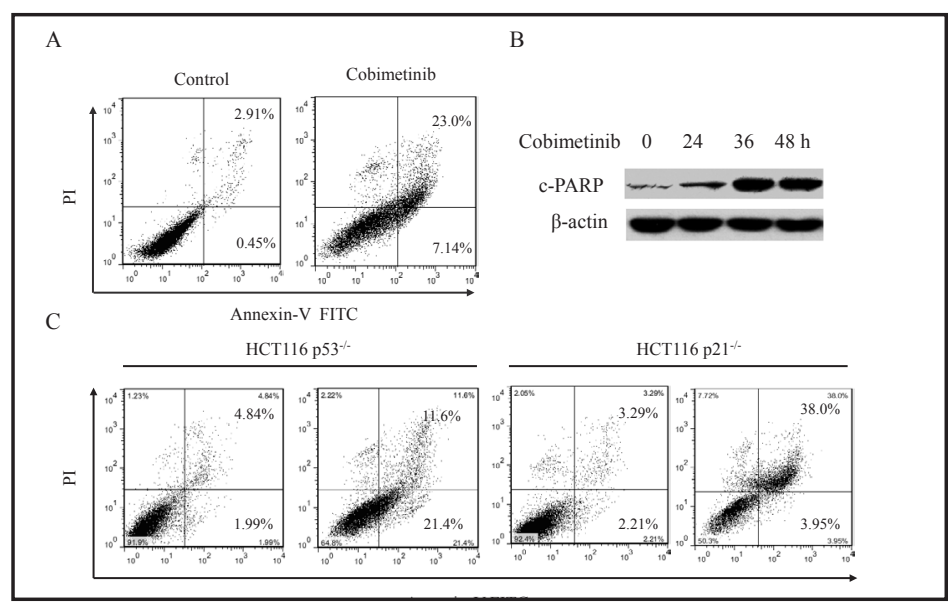

Fig. 5. Comparative analysis of transcriptome data and GEO data. (A) Number of downregulated and upregulated DEGs; upper panel, cobimetinib treatment vs. control; lower panel, colorectal cancer vs. normal tissues). (B) According to $\mathrm{p}$-values, the top 20 enriched pathways for DEGs (upper panel, the pathways of cobimetinib vs. control; lower panel, pathways of colorectal cancer vs. normal tissues). (C) Venn diagrams showing the overlapping DEGs between transcriptome data and GEO data. Upper diagram, 313 genes highly expressed in colorectal cancer tissues were downregulated by cobimetinib in HCT116 cells (green, 1,406 downregulated DEGs after cobimetinib treatment in HCT116; red, 2,125 upregulated DEGs in colorectal cancer tissues compared to normal tissues). Lower diagram, 448 genes with lower expression in colorectal cancer tissues were upregulated by cobimetinib in

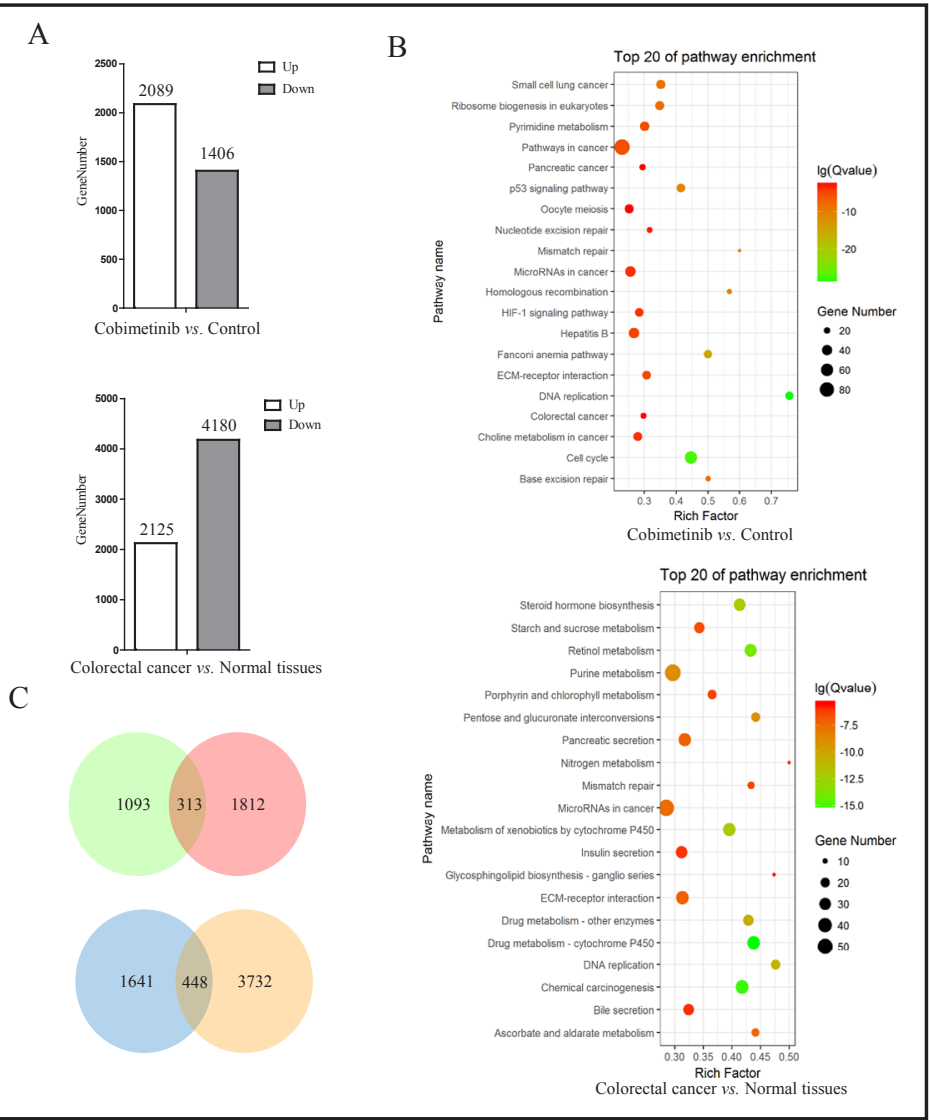
HCT116 cells (blue, 2,089 upregulated DEGs after cobimetinib treatment in HCT116; orange, 4,180 downregulated DEGs in colorectal cancer tissues compared to normal tissues).

\section{KARGER}


the transcriptomes were sequenced. We found that expression patterns of HCT116 after cobimetinib treatment for 24 or $36 \mathrm{~h}$ showed the same trend. Therefore, RNA-seq data after cobimetinib treatment for $24 \mathrm{~h}$ was used for the next study. After cobimetinib treatment for $24 \mathrm{~h}, 3,495$ differentially expressed genes (DEGs) were found, including 2, 089 upregulated genes and 1, 406 downregulated genes (Fig. 5A, upper panel), and most of these DEGs were mapped to the DNA replication, cell cycle, Fanconi anemia, p53 signaling, mismatch repair, and base excision repair pathways (Fig. 5B).

We downloaded the data set corresponding to GEO series accession number GSE21815 $[17,18]$ from the GEO database, which includes gene expression profiles of 132 colorectal cancer tissues and nine normal human intestinal epithelial tissues. DEGs between normal tissues and tumors were analyzed using GeneSpring software. Finally, by comparisons with normal tissues, 6, 305 DEGs were obtained from among colorectal tumors, including 2, 125 upregulated genes and 4, 180 downregulated genes (Fig. 5A, lower panel; Table, 2). G0 and KEGG analyses showed that these DEGs were enriched in the pathways of DNA replication, mismatch repair, drug metabolism-other enzymes, drug metabolism-cytochrome P450, and pentose and glucuronate interconversions (Fig. 5B).

To further determine the key modulators for cobimetinib treatment, a comparative analysis was performed between the RNA-seq and GEO datasets. We found that a total of 313 genes that were highly expressed in colorectal cancer tissues were downregulated by cobimetinib in HCT116 cells (Fig. 5C, upper), including CCND1, E2F1, CDC25C, CCNE2, MYC, and PCNA. Furthermore, 448 genes with lower expression in colorectal cancer tissues were upregulated by cobimetinib treatment in HCT116 cells (Fig. 5C, lower panel), including PRKCA, PI3K, RTK, and PKC. Then, we conducted a KEGG pathway enrichment analysis for these up- and downregulated genes. Table 3 shows the statistically significant KEGG pathways and the correlated DEGs (Table 3). The results of overlap and pathway enrichment analysis revealed that PKC and PI3K pathways were activated after cobimetinib treatment.

Inhibition of the PI3K or PKC pathway could increase cobimetinib cytotoxicity in HCT116 cells

Through bioinformatics analyses, we found that PI3K and PKC pathways were activated after cobimetinib treatment. Activation of the PI3K or PKC pathway has been reported to be associated with tumorigenesis [19-21]. Therefore, activation of the PI3K and PKC pathways may attenuate the effects of cobimetinib. To explore the possibility of combination therapy with other inhibitors, we used Go 6983 (a Pan PKC inhibitor) and VS-5584 (a PI3K-mTOR dual inhibitor) alone or in combination with cobimetinib to treat HCT116 cells. MTT assays showed that both Go 6983 and VS-5584 can inhibit the viability of HCT116 cells and also have synergistic effects with cobimetinib (with a CI of less than 1) in HCT116 cells (Fig. 6A and B). Furthermore, treatment with Go 6983 or VS-5584 alone induced moderate G1 arrest and apoptosis in HCT116 cells, and Go 6983 or VS-584 significantly induced cell cycle arrest and apoptosis in combination with cobimetinib (Fig. 6C and D). These results revealed that inhibition of the PI3K or PKC pathway could increase the cytotoxicity of cobimetinib in HCT116 cells.

Cobimetinib enhances 5-FU cytotoxicity by decreasing TYMS expression in HCT116 cells

Intriguingly, we found that thymidylate synthetase (TYMS), an important direct target of

Table 2. Top 10 most significant differentially expressed genes upregulated and downregulated in colorectal cancer tissues compared with normal tissues

\begin{tabular}{llll}
\hline \multicolumn{2}{l}{ Upregulated genes } & \multicolumn{2}{l}{ Downregulated genes } \\
\hline Gene & log FC & Gene & \multicolumn{1}{l}{$\log$ FC } \\
TMEM97 & -1.149511 & ZG16 & 9.3678875 \\
SP5 & -2.9767313 & CA1 & 8.558522 \\
HSPD1 & -1.1093872 & GCG & 7.6726413 \\
PAQR4 & -1.1977377 & CLCA1 & 7.436072 \\
IL2RA & -1.2920417 & SLC4A4 & 7.203979 \\
DYNLT3 & -1.7005748 & ITLN1 & 6.9722238 \\
POLE2 & -1.2588199 & CLCA4 & 6.8729124 \\
CCND1 & -1.3114972 & GUCA2B & 6.8429737 \\
BRCA2 & -1.4148383 & CA4 & 6.5177035 \\
CENPN & -1.3245826 & CA2 & 6.4782157 \\
\hline
\end{tabular}




\begin{tabular}{|c|c|c|}
\hline \multirow{2}{*}{$\begin{array}{c}\text { Cellular Physiology } \\
\text { and Biochemistry }\end{array}$} & \multicolumn{2}{|c|}{ Cell Physiol Biochem 2018:47:680-693 } \\
\hline & $\begin{array}{l}\text { DOI: } 10.11159 / 000490022 \\
\text { Published online: May } 25,2018\end{array}$ & $\begin{array}{l}\text { O } 2018 \text { The Author(s). Published by S. Karger AG, Basel } \\
\text { www.karger.com/cpb }\end{array}$ \\
\hline
\end{tabular}

Table 3. Top five statistically significant KEGG pathways and correlated DEGs among colorectal cancer tissues. Note: The top half of the table shows the top five statistically significant KEGG pathways enriched among 448 upregulated DEGs in colorectal cancer tissues compared to normal tissues. The lower half of the table shows the top five statistically significant KEGG pathways enriched among 313 downregulated DEGs in colorectal cancer tissues compared to normal tissues

\begin{tabular}{|c|c|c|c|c|c|}
\hline & $\begin{array}{l}\text { Pathway } \\
\text { ID }\end{array}$ & Pathways & $\begin{array}{l}\text { Gene } \\
\text { Counts }\end{array}$ & $P$ value & Genes \\
\hline \multirow{5}{*}{ 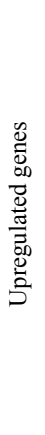 } & ko05032 & Morphine addiction & 7 & 0.002918 & PRKCA, ADCY9, PDE3A, PDE8B, PDE4D, GNG7, GNG13 \\
\hline & ko04713 & Circadian entrainment & 7 & 0.003085 & $\begin{array}{l}\text { PRKCA, ADCY9, CAMK2B, PRKG1, CACNA1I, GNG7, } \\
\text { GNG13 }\end{array}$ \\
\hline & ko04360 & Axon guidance & 8 & 0.004132 & $\begin{array}{l}\text { SEMA3B, CXCR4, UNC5B, SEMA6A, EPHA4, SEMA4G, } \\
\text { SLIT2, ROBO3, }\end{array}$ \\
\hline & ko04151 & $\begin{array}{l}\text { PI3K-Akt signaling } \\
\text { pathway }\end{array}$ & 15 & 0.004952 & $\begin{array}{l}\text { SGK2, THBS1, PRKCA, CHAD, ITGB7, FGFR2, EPOR, } \\
\text { PIK3R5, COL4A5, COL4A6, NR4A1, FGFR3, GNG13, } \\
\text { LAMA3, GNG7, }\end{array}$ \\
\hline & ko04810 & $\begin{array}{l}\text { Regulation of actin } \\
\text { cytoskeleton }\end{array}$ & 11 & 0.005664 & $\begin{array}{l}\text { ACTN2, SLC9A1, ITGB7, SCIN, NCKAP1L, FGFR2, } \\
\text { PIK3R5, ITGAX, FGFR3, IQGAP2, GSN }\end{array}$ \\
\hline \multirow{5}{*}{ 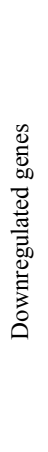 } & ko04110 & Cell cycle & 22 & $1.31 \mathrm{E}-15$ & $\begin{array}{l}\text { CCND1, RBL1, CHEK1, ORC6, RB1, E2F1, CDC25C, } \\
\text { MAD2L1, CCNE2, PLK1, MYC, BUB3, PCNA, ORC3, } \\
\text { MCM4, CCNA2, CDC6, BUB1, CDK1, CCNB1, TTK, } \\
\text { TFDP1 }\end{array}$ \\
\hline & ko03030 & DNA replication & 12 & $2.47 \mathrm{E}-12$ & $\begin{array}{l}\text { PRIM1, RNASEH2A, RPA3, PCNA, POLE2, PRIM2, RFC5, } \\
\text { MCM4, RFC2, RFC3, RFC4, RNASEH1 }\end{array}$ \\
\hline & ko03430 & Mismatch repair & 7 & $5.05 \mathrm{E}-07$ & RPA3, PCNA, EXO1, RFC5, RFC2, RFC3, RFC4 \\
\hline & ko04914 & $\begin{array}{l}\text { Progesterone-mediated } \\
\text { oocyte maturation }\end{array}$ & 9 & 4.21E-05 & $\begin{array}{l}\text { HSP90AA1, HSP90AB1, CDK1, CCNA2, CDC25C, } \\
\text { MAD2L1, PLK1, BUB1, CCNB1 }\end{array}$ \\
\hline & ko03420 & Nucleotide excision repair & 7 & 0.000202 & RPA3, PCNA, POLE2, RFC5, RFC2, RFC3, RFC4 \\
\hline
\end{tabular}

5-Florouracil (5-FU), was significantly downregulated by cobimetinib through bioinformatic analyses. TYMS plays a key role in DNA synthesis and its high expression is responsible for the 5-FU resistance of colorectal cancer [22, 23]. qRT-PCR and western blot analysis showed that both mRNA and protein levels of TYMS decreased significantly after cobimetinib treatment in HCT116 cells ( $\mathrm{p}<0.01$ ) (Fig. 7A). Therefore, we hypothesized that cobimetinib could enhance the cytotoxicity of 5-FU in HCT116 cells. To explore the potential synergy between cobimetinib and 5-FU, we evaluated effects of single and combination treatment with cobimetinib and 5-FU in HCT116 cells by MTT assay. Cobimetinib was thus revealed to act synergistically with 5-FU, with a CI of less than 1 (Fig. 7B). In order to further explore their combined effects, we examined the cell cycle by using flow cytometry. As shown in Fig. 7C, combination treatment with cobimetinib and 5-FU showed more G1 arrest than treatment with cobimetinib or 5-FU alone. These results suggest that cobimetinib could enhance 5-FU cytotoxicity in HCT116 cells.

\section{Discussion}

Cobimetinib is a drug marketed for treating BRAF-mutated melanoma. Previous studies have shown that cobimetinib can inhibit the cell viability of many cancer cells, such as hepatocellular carcinoma [16, 24]. In this study, we found that cobimetinib could inhibit cell 


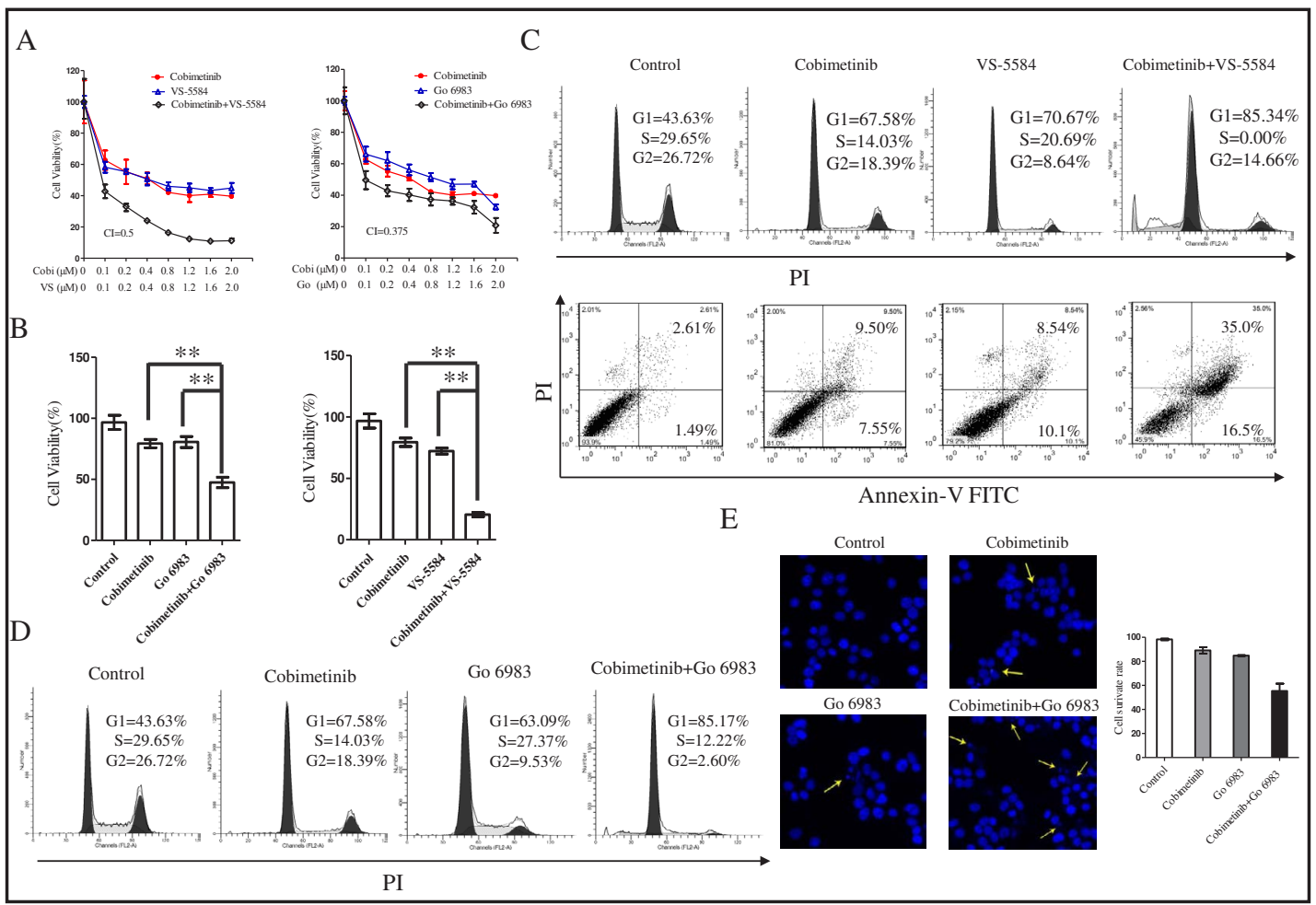

Fig. 6. Go 6983 and VS-5584 increased the cytotoxicity of cobimetinib in HCT116 cells. (A) HCT116 cells were treated with cobimetinib or 5-FU alone or with cobimetinib and 5-FU in combination at indicated concentrations for $48 \mathrm{~h}$. Cell viability was evaluated by MTT assays. Error bars represent means \pm SD. (B) HCT116 cells were treated with Go $6983(0.75 \mu \mathrm{M})$ or VS-5584 $(0.6 \mu \mathrm{M})$ alone or in combination with cobimetinib $(0.15 \mu \mathrm{M})$ for $48 \mathrm{~h}$. Then, cell viability was evaluated by MTT (C, D, and E) HCT116 cells were treated with Go $6983(0.75 \mu \mathrm{M})$ or VS-5584 $(0.6 \mu \mathrm{M})$ alone or in combination with cobimetinib $(0.15 \mu \mathrm{M})$ for $48 \mathrm{~h}$. Then, cell cycle arrest was determined by flow cytometry, and cell apoptosis was determined by flow cytometry or Hoechst 33258 staining. ${ }^{*} \mathrm{p}<0.01$.

Fig. 7. Cobimetinib enhances $5-\mathrm{FU}$ cytotoxicity in HCT116 cells. (A) Protein and mRNA levels of TYMS after cobimetinib $(1 \mu \mathrm{M})$ treatment. (B) HCT116 cells were treated with cobimetinib or 5-FU alone or with cobimetinib and 5-FU in combination at indicated concentrations for $48 \mathrm{~h}$. Cell viability was evaluated by MTT assay. Error bars represent means \pm SD. (C) HCT116 cells were treated with cobimetinib $(0.05 \mu \mathrm{M})$ or 5 -FU $(2.5 \mu \mathrm{M})$ alone or with cobimetinib $(0.05 \mu \mathrm{M})$ and 5 -FU $(2.5 \mu \mathrm{M})$ in combination for $48 \mathrm{~h}$, and then the cell cycle distributions were determined by flow cytometry. ${ }^{*} \mathrm{p}<0.01$.

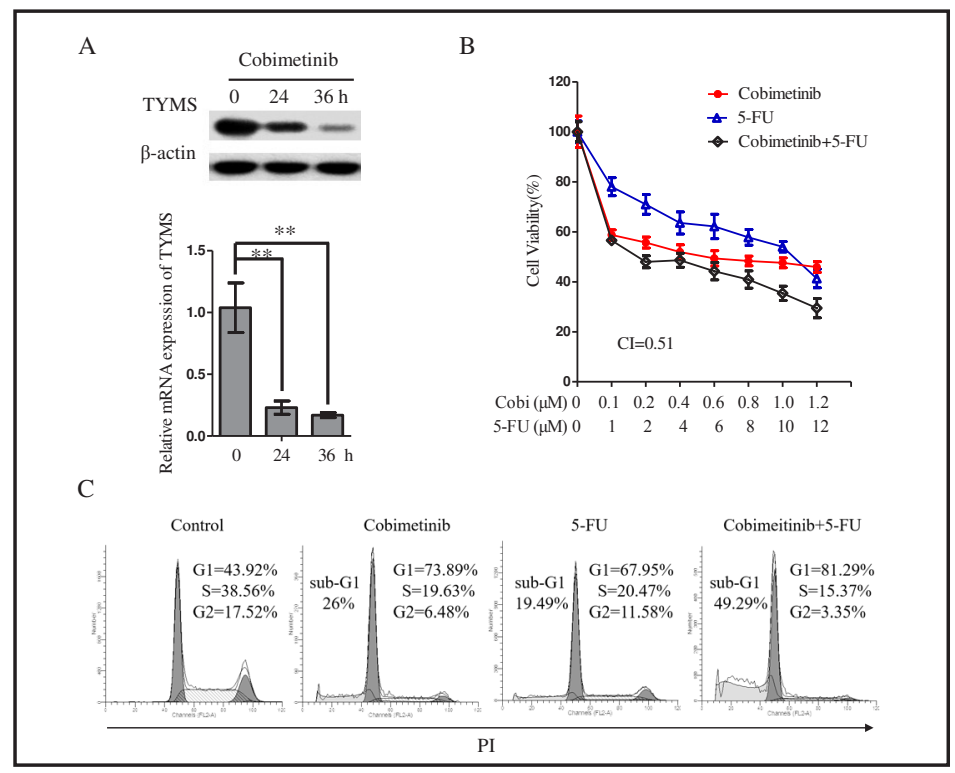


viability by inducing G1 cell arrest and apoptosis in HCT116 colorectal cancer cells. Moreover, G1 arrest induced by cobimetinib was independent of p53 or p21. We also found that cobimetinib may induce G1 arrest via downregulation of cell cycle related genes, including CCND1, E2F1, CDC25C, CCNE2, MYC, and PCNA. CCND1 and CDC25C are downstream of MEK/ERK. ERK can regulate cyclin D1 transcriptional induction via Fos family members and MYC [25-27], and cyclin D1 can promote phosphorylation and inactivation of Rb, which then activates E2F1 and induces cyclin E expression [28]. In addition, ERK also regulates the assembly of the cyclin/CDK complex [25].

In order to further understand the mechanisms of cobimetinib, we performed RNAseq to identify DEGs between treatments with and without cobimetinib in HCT116 cells and compared these DEGs with the GEO dataset from colorectal cancer tissues and normal colonic epithelial tissues. Finally, we found that PKC and PI3K/Akt pathways were activated after cobimetinib treatment.

PKC, a family of specific serine/threonine kinases, is involved in different cellular processes via target protein phosphorylation [29]. PKC $\alpha$ is expressed in many tissues, particularly in transformed cells, and plays a key role in the process of cell carcinogenesis and tumor angiogenesis. PKC $\alpha$ can regulate cell division and promote cancer cell growth. Previous studies have shown that inhibition of PKC $\alpha$ can decrease cell proliferation and migration and that interfering with the expression of PKC $\alpha$ by siRNA can reduce multidrug resistance in cancer cells and restore their sensitivity to anticancer drugs [30, 31]. The effects of PKC $\alpha$ on cancer cells is related not only to the cell types but also to the conditions [32]. It has been reported that PKC $\alpha$ could inhibit the occurrence of intestinal cancer and the expression level of PKC $\alpha$ in colorectal cancer cells is low relative to other cancers [33, 34]. In contrast, some studies have shown that PKC $\alpha$ activation can promote cancer cell proliferation, migration, survival, and drug resistance [35-37]. In colorectal cancer, the PKC $\alpha$ inhibitor can reduce cell viability and inhibit cell migration [38]. In this study, we showed that the PKC $\alpha$ inhibitor Go 6983 could enhance the cytotoxicity of cobimetinib in HCT116 cells.

The PI3K/Akt pathway plays an important role in many cellular processes, such as cell survival, proliferation, migration, differentiation, and tumorigenesis [39-41]. Several studies have indicated that aberrant activation of the PI3K/Akt pathway is found in many human cancers, including colorectal cancer [20,42]. Accordingly, the PI3K/Akt pathway is an ideal target for cancer therapy [41]. At present, the PI3K/Akt inhibitor has been applied to cancer therapy in preclinical trials $[41,43,44]$. In this study, the results of RNA-seq and GEO data revealed that the PI3K/Akt pathway was activated after cobimetinib treatment of HCT116 cells. Furthermore, the PI3K inhibitor VS-5584 can enhance the cytotoxicity of cobimetinib in HCT116 cells. Thus, inhibition of PKC and PI3K/Akt pathways is an effective method for enhancing the efficacy of cobimetinib in colorectal cancer cells.

5-FU is widely used in the treatment of many cancers, especially colorectal cancer. TYMS is a downstream target molecule of 5-FU and plays a key role in DNA synthesis. TYMS can catalyze the methylation of deoxyuridine monophosphate to produce deoxythymidine triphosphate, followed by synthesis of thymidylate [45]. It has been reported that the high expression of TYMS is responsible for resistance to 5-FU in patients with colorectal cancers $[46,47]$. Moreover, high TYMS expression levels is a sign of poor prognosis in colorectal cancer $[48,49]$. Intriguingly, we found that cobimetinib can significantly decrease TYMS expression in both mRNA and protein levels and enhance the efficacy of 5-FU. In addition, our results also suggest that cobimetinib can act synergistically with 5-FU.

Taken together, our data showed that cobimetinib not only inhibited cell proliferation but also induced G1 phase arrest and apoptosis in colorectal cancer cells HCT116. Furthermore, inhibition of the PKC or PI3K pathway can increase the cytotoxicity of cobimetinib in HCT116 cells. Notably, we found that cobimetinib could enhance the efficacy of 5-FU by decreasing TYMS expression. Considering the use of cobimetinib as a clinical drug and its synergistic action with 5-FU, our results suggest the potential utility of cobimetinib in colorectal cancer therapy. 


\section{Cellular Physiology Cell Physiol Biochem 2018;47:680-693 \\ \begin{tabular}{l|l} 
DOI: 10.1159/000490022 & O 2018 The Author(s). Published by S. Karger AG, Basel \\
www.karger.com/cpb
\end{tabular}}

Gong et al.: Potential of Cobimetinib in Colorectal Cancer

\section{Acknowledgements}

This research was supported by the National Natural Science Foundation of China (81672942) and Chengdu Municipal Science and Technology Bureau (2015-HM01-00071SF).

\section{Disclosure Statement}

The authors declare that they have no conflict of interests.

\section{References}

1 Manousaridis I, Mavridou S, Goerdt S, Leverkus M, Utikal J: Cutaneous side effects of inhibitors of the RAS/ RAF/MEK/ERK signalling pathway and their management. J Eur Acad Dermatol Venereol 2013;27:11-18.

2 Chappell WH, Steelman LS, Long JM, Kempf RC, Abrams SL, Franklin RA, Bäsecke J, Stivala F, Donia M, Fagone P: Ras/Raf/MEK/ERK and PI3K/PTEN/Akt/mTOR inhibitors: rationale and importance to inhibiting these pathways in human health. Oncotarget 2011;2:135-164.

- 3 Vakana E, Pratt S, Blosser W, Dowless M, Simpson N, Yuan XJ, Jaken S, Manro J, Stephens J, Zhang Y: LY3009120, a panRAF inhibitor, has significant anti-tumor activity in BRAF and KRAS mutant preclinical models of colorectal cancer. Oncotarget 2017;8:9251-9266.

-4 Ren J, Li G, Ge J, Li X, Zhao Y: Is K-ras gene mutation a prognostic factor for colorectal cancer: a systematic review and meta-analysis. Dis Colon Rectum 2012;55:913-923.

5 Nandan MO, Yang VW: An update on the biology of RAS/RAF mutations in colorectal cancer. Curr Colorectal Cancer Rep 2011;7:113-120.

6 Gallagher DJ, Kemeny N: Metastatic Colorectal Cancer: From Improved Survival to Potential Cure. Oncology 2010;78:237-248.

7 Wilhelm SM, Adnane L, Newell P, Villanueva A, Llovet JM, Lynch M: Preclinical overview of sorafenib, a multikinase inhibitor that targets both Raf and VEGF and PDGF receptor tyrosine kinase signaling. Mol Cancer Ther 2008;7:3129-3140.

8 Chen D, Wei L, Yu J, Zhang L: Regorafenib inhibits colorectal tumor growth through PUMA-mediated apoptosis. Clin Cancer Res 2014;20:3472-3484.

-9arnock-Jones KP: Cobimetinib: First Global Approval. Drugs 2015;75:1823-1830.

10 Medina TM, Lewis KD: The evolution of combined molecular targeted therapies to advance the therapeutic efficacy in melanoma: a highlight of vemurafenib and cobimetinib. Onco Targets Ther 2016;9:3739-3752.

11 Kawakami H, Huang S, Pal K, Dutta SK, Mukhopadhyay D, Sinicrope FA: Mutant BRAF upregulates MCL-1 to confer apoptosis resistance that is reversed by MCL-1 antagonism and cobimetinib in colorectal cancer. Mol Cancer Ther 2016;15:3015-3027

12 Sriraman SK, Geraldo V, Luther E, Degterev A, Torchilin V: Cytotoxicity of PEGylated liposomes co-loaded with novel pro-apoptotic drug NCL-240 and the MEK inhibitor cobimetinib against colon carcinoma in vitro. J Control Release 2015;220:160-168.

13 Bendell JC, Kim TW, Goh BC, Wallin J, Oh D-Y, Han S-W, Lee CB, Hellmann MD, Desai J, Lewin JH, Solomon BJ, Chow LQM, Miller WH, Gainor JF, Flaherty K, Infante JR, Das-Thakur M, Foster P, Cha E, Bang Y-J: Clinical activity and safety of cobimetinib (cobi) and atezolizumab in colorectal cancer (CRC). J Clin Oncol 2016;34:3502-3502.

14 Chen D, Ming L, Zou F, Peng Y, Van HB, Yu J, Zhang L: TAp73 promotes cell survival upon genotoxic stress by inhibiting p53 activity. Oncotarget 2014;5:8107-8122.

15 Chou TC: Drug combination studies and their synergy quantification using the Chou-Talalay method. Cancer Res 2010;70:440-446.

-16 Singh A, Ruan Y, Tippett T, Narendran A: Targeted inhibition of MEK1 by cobimetinib leads to differentiation and apoptosis in neuroblastoma cells. J Exp Clin Cancer Res 2015;34:104-116. 


\section{Cellular Physiology Cell Physiol Biochem 2018;47:680-693 \begin{tabular}{l|l|l} 
DOI: 10.1159/000490022 & O 2018 The Author(s). Published by S. Karger AG, Basel \\
www.karger.com/cpb
\end{tabular}}

Gong et al.: Potential of Cobimetinib in Colorectal Cancer

17 Kogo R, Shimamura T, Mimori K, Kawahara K, Imoto S, Sudo T, Tanaka F, Shibata K, Suzuki A, Komune S, Miyano S, Mori M: Long noncoding RNA HOTAIR regulates polycomb-dependent chromatin modification and is associated with poor prognosis in colorectal cancers. Cancer Res 2011;71:6320-6326.

18 Iwaya T, Yokobori T, Nishida N, Kogo R, Sudo T, Tanaka F, Shibata K, Sawada G, Takahashi Y, Ishibashi M: Downregulation of miR-144 is associated with colorectal cancer progression via activation of mTOR signaling pathway. Carcinogenesis 2012;33:2391-2397.

19 Chen J, Shao R, Li F, Monteiro M, Liu JP, Xu ZP, Gu W: PI3K/Akt/mTOR pathway dual inhibitor BEZ235 suppresses the stemness of colon cancer stem cells. Clin Exp Pharmacol Physiol 2016;42:1317-1326.

20 Danielsen SA, Eide PW, Nesbakken A, Guren T, Leithe E, Lothe RA: Portrait of the PI3K/AKT pathway in colorectal cancer. Biochim Biophys Acta 2015;1855:104-121.

-21 Garg R, Blando JM, Perez CJ, Abba MC, Benavides F, Kazanietz MG: Protein Kinase C Epsilon Cooperates with Pten Loss for Prostate Tumorigenesis Through the Cxcl13-Cxcr5 Pathway. Cell Rep 2017;19:375-388.

22 Peters GJ, van Groeningen CJ: Clinical relevance of biochemical modulation of 5-fluorouracil. Ann Oncol 1991;2:469-480.

23 Peters GJ, Backus HHJ, Freemantle S, Triest BV, Codacci-Pisanelli G, Wilt CLVD, Smid K, Lunec J, Calvert AH, Marsh S: Induction of thymidylate synthase as a 5-fluorouracil resistance mechanism. Biochim Biophys Acta 2002;1587:194-205.

24 Gong S, Xu D, Zou F, Peng R: (-)-Curine induces cell cycle arrest and cell death in hepatocellular carcinoma cells in a p53-independent way. Biomed Pharmacother 2017;89:894-901.

-25 Chambard JC, Lefloch R, Pouysségur J, Lenormand P: ERK implication in cell cycle regulation. Biochim Biophys Acta 2007;1773:1299-1310.

26 Chang F, Steelman LS, Shelton JG, Lee JT, Navolanic PM, Blalock WL, Franklin R, Mccubrey JA: Regulation of cell cycle progression and apoptosis by the Ras/Raf/MEK/ERK pathway (Review). Int J Oncol 2003;22:469480 .

-27 Mccubrey JA, Steelman LS, Chappell WH, Abrams SL, Wong EW, Chang F, Lehmann B, Terrian DM, Milella M, Tafuri A: Roles of the Raf/MEK/ERK pathway in cell growth, malignant transformation and drug resistance. Biochim Biophys Acta 2007;1773:1263-1284.

28 Torii S, Yamamoto T, Tsuchiya Y, Nishida E: ERK MAP kinase in G 1 cell cycle progression and cancer. Cancer Sci 2006;97:697-702.

29 Nishizuka Y: Studies and Perspectives of Protein Kinase C. Science 1986;233:305-312.

-30 Zhao LJ, Xu H, Qu JW, Zhao WZ, Zhao YB, Wang JH: Modulation of drug resistance in ovarian cancer cells by inhibition of protein kinase C-alpha (PKC- $\alpha$ ) with small interference RNA (siRNA) agents. Asian Pac J Cancer Prev 2012;13:3631-3636.

-31 Wu TT, Hsieh YH, Hsieh YS, Liu JY: Reduction of PKC alpha decreases cell proliferation, migration, and invasion of human malignant hepatocellular carcinoma. J Cell Biochem 2008;103:9-20.

32 Nakashima S: Protein kinase C alpha (PKC alpha): regulation and biological function. J Biochem 2002;132:669-675.

-33 Oster H, Leitges M: Protein kinase C $\alpha$ but not PKC 3 suppresses intestinal tumor formation in ApcMin/+ mice. Cancer Res 2006;66:6955-6963.

-34 Konopatskaya 0, Poole AW: Protein kinase C $\alpha$ : disease regulator and therapeutic target. Trends Pharmacol Sci 2010;31:8-14.

-35 Shimatsu T, Takahashi M, Uwazumi H, Sato T, Takahashi M, Wakiyama T: Protein kinase C $\alpha$ protects against multidrug resistance in human colon cancer cells. Mol Cells 2012;34:61-69.

-36 Ghoul A, Serova M, Astorgues-Xerri L, Bieche I, Bousquet G, Varna M, Vidaud M, Phillips E, Weill S, Benhadji KA: Epithelial-to-mesenchymal transition and resistance to ingenol 3-angelate, a novel protein kinase $\mathrm{C}$ modulator, in colon cancer cells. Cancer Res 2009;69:4260-4269.

37 Wu B, Zhou H, Hu L, Mu Y, Wu Y: Involvement of PKC $\alpha$ activation in TF/VIIa/PAR2-induced proliferation, migration, and survival of colon cancer cell SW620 Tumour Biol 2013;34:837-846.

38 Masur K, Lang K, Niggemann B, Zanker KS, Entschladen F: High PKC alpha and low E-cadherin expression contribute to high migratory activity of colon carcinoma cells. Mol Biol Cell 2001;12:1973-1982.

39 Cantley LC: The phosphoinositide 3-kinase pathway. Science 2002;296:1655-1657.

40 Janku F: Phosphoinositide 3-kinase (PI3K) pathway inhibitors in solid tumors: From laboratory to patients. Cancer Treat Rev 2017;59:93-101. 


\section{Cellular Physiology Cell Physiol Biochem 2018;47:680-693

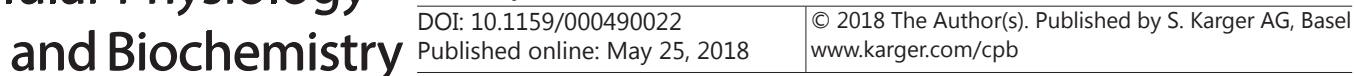 \\ Gong et al.: Potential of Cobimetinib in Colorectal Cancer}

41 Ponstostivint E, Thibault B, Guillermetguibert J: Targeting PI3K Signaling in Combination Cancer Therapy. Trends Cancer 2017;3:454-469.

42 Ghayad SE, Cohen PA: Inhibitors of the PI3K/Akt/mTOR pathway: new hope for breast cancer patients. Recent Pat Anticancer Drug Discov 2010;5:29-57.

-43 Mcneill RS, Canoutas DA, Stuhlmiller TJ, Dhruv HD, Irvin DM, Bash RE, Angus SP, Herring LE, Simon JM, Skinner KR: Combination therapy with potent PI3K and MAPK inhibitors overcomes adaptive kinome resistance to single agents in preclinical models of glioblastoma. Neuro Oncol 2017;19:1469-1480.

44 Aed VS, Sambade MJ, Siegel MB, Sud S, Mcneill RS, Bevill SM, Chen X, Bash RE, Mounsey L, Golitz BT: Combined kinase inhibitors of MEK1/2 and either PI3K or PDGFR are efficacious in intracranial triplenegative breast cancer. Neuro Oncol 2017;19:1481-1493.

45 Hua D, Huang ZH, Mao Y, Deng JZ: Thymidylate synthase and thymidine phosphorylase gene expression as predictive parameters for the efficacy of 5-fluorouracil-based adjuvant chemotherapy for gastric cancer. World J Gastroenterol 2007;13:5030-5034.

46 Gotanda K, Hirota T, Matsumoto N, Ieiri I: MicroRNA-433 negatively regulates the expression of thymidylate synthase (TYMS) responsible for 5-fluorouracil sensitivity in HeLa cells. BMC Cancer 2013;13:369-376.

47 Xu W, Jiang H, Zhang F, Gao J, Hou J: MicroRNA-330 inhibited cell proliferation and enhanced chemosensitivity to 5 -fluorouracil in colorectal cancer by directly targeting thymidylate synthase. Oncol Lett 2017;13:3387-3394.

48 Mekenkamp LJ, Heesterbeek KJ, Koopman M, Tol J, Teerenstra S, Venderbosch S, Punt CJ, Nagtegaal ID: Mucinous adenocarcinomas: poor prognosis in metastatic colorectal cancer. Eur J Cancer 2012;48:501-509.

49 Lu Y, Zhuo C, Cui B, Liu Z, Zhou P, Lu Y, Wang B: TYMS serves as a prognostic indicator to predict the lymph node metastasis in Chinese patients with colorectal cancer. Clin Biochem 2013;46:1478-1483. 